\title{
WHAT DO MIRA VARIABLES TELL US ABOUT THE LATE EVOLUTION OF LOW MASS STARS?
}

\author{
M.W. Feast
}

South African Astronomical Observatory

P.O. Box 9, Observatory 7935, Cape, South Africa.

(1) Mira variables define the tip of the AGB for low mass stars (cf. Feast and Whitelock 1987 fig. 1).

(2) The kinematics of Mira variables are a function of period (e.g. Feast 1989 and earlier references) and for Miras in globular clusters the period is a function of metallicity (e.g. Feast 1981). Hence the Mira period sequence is not an evolutionary sequence but represents the sequence of AGB tips for stars of different initial mass and/or chemical composition and/or age.

(3) Mira are surrounded by circumstellar dust shells. The mass loss rates are a function of period and light amplitude (Whitelock et al. 1987, Whitelock et al. 1991, Whitelock 1990). The results suggest evolution with increasing pulsation amplitude and mass loss but little change in $\log P((2)$ and (3) do not preclude some (small) change in $\log P$ during evolution nor possible phases of decreasing light amplitude and mass loss).

(4) Mass loss seems likely to be due to atmospheric extention produced by pulsation-driven shock waves followed by dust formation and expulsion by radiation pressure (e.g. Wood 1979). Alternative mechanisms are acoustic waves (Pijpers and Hearn 1989, Pijpers and Habing 1989) or Alfven waves (Hartmann and MacGregor 1980). Whatever the mechanism the results show that the energy input into the mass loss increases with pulsation amplitude at a given period.

(5) Mass loss rates range from $\sim 10^{-7} \mathrm{M}_{\odot} \mathrm{yr}^{-1}$ to $\sim 3 \times 10^{-5} \mathrm{M}_{\odot} \mathrm{yr}^{-1}$ (and to $\sim 10^{-4} \mathrm{M}_{\odot} \mathrm{y} \mathrm{r}^{-1}$ for extreme OH/IR Miras) (cf. Whitelock 1990). Mira lifetimes have been estimated as $\sim 3 \times 10^{5}$ yr for Miras in globular clusters (Renzini and Greggio 1990) or 5x104 yr from PN/Mira ratios (Wood 1990). These figures suggest that there is sufficient mass loss in the Mira phase to form the subsequent planetary nebula. 
(6) Miras in the LMC show PL and PLC relations. The temperature term in the PL $T_{\text {eff }}$ relation is $\sim-10 \log \mathrm{T}_{\text {eff. }}$ This implies that to a first approximation the pulsation mass is a function of period (Feast et al. 1989).

(7) Amongst the uncertainties in interpreting Mira observations are uncertainties in calibrating their $T_{\text {eff }}$ values and the possible effects of mass loss on pulsation period (Pijpers 1991).

(8) Wood et al. (1991) combine a theoretical pulsation equation with a theoretical AGB relation to obtain:

$\mathrm{M}_{\text {bol }}=-1.57 \log \mathrm{P}+0.73 \log \mathrm{z}-2.45 \log \mathrm{M}-6.1 \Delta+$ const:

$\log \mathrm{T}_{\text {eff }}=-0.10 \log \mathrm{P}-0.072 \log \mathrm{z}+0.013 \log \mathrm{M}+0.61 \Delta+$ const: $(\mathrm{J}-\mathrm{K})=+0.50 \log \mathrm{P}+0.36 \log \mathrm{z}-0.065 \log \mathrm{M}-3.05 \Delta+$ const:

( $\mathrm{M}=$ mass, $\mathrm{z}=$ metal abundance). $\quad \Delta$ is zero for little mass loss and becomes significant when the evolutionary tracks begin to bend back towards higher temperatures as the stars move off the AGB.

(9) An evolutionary track is: $M_{\text {bol }}=-1.57 \log P+$ const (i.e. $M \sim$ const, $z \sim$ const, $\Delta \sim 0)$. This is close to the Whitelock relation for SR variables in globular clusters (Whitelock 1986, Feast 1989) which, for metal-rich clusters, is: $\mathrm{M}_{\text {bol }}=-1.34 \log \mathrm{P}+$ const. These $\mathrm{SR}$ variables are indeed believed to lie on an evolutionary track leading to Miras. If there is mild mass loss in the SR phase $\left(\sim 10^{-7} \mathrm{M}_{\odot} \mathrm{yr}^{-1}\right)$ then exact agreement between theory and observation can be achieved. The Mira PL relation, $\mathrm{M}_{\mathrm{bol}}=-3.00 \log \mathrm{P}+$ const, (Feast et al. 1989) is too steep for an evolutionary track (cf. (2) and (3) above).

(10) Application of equations (1) and (2) (or similar relations) to SR variables in globular clusters of different metallicities suggests that metal rich $(\log z>-0.7)$ and metal poor SR variables are pulsating in different modes (Whitelock 1986, Perl and Tuchman 1990).

(11) The $(\mathrm{J}-\mathrm{K})-\log \mathrm{P}$ relation for Miras is closely similar in a variety of locations (Whitelock et al. 1991 Figs 8 and 9). For equation (2b) to hold, the $\mathrm{P}-\mathrm{z}$ relation must then be closely the same everywhere. This may mean that all Miras are of the same (old) age. The initial mass would then be a function of metallicity for an evolved star (VandenBerg and Laskarides 1987) and this could lead to a current mass-metallicity relation.

(12) Including recent data, the dependence of period on metallicity for Miras in galactic globular clusters is $\log z=3.6 \log P+$ const. Combined with (2b) this gives: 


$$
(\mathrm{J}-\mathrm{K})=1.8 \log \mathrm{P}-0.065 \log \mathrm{M}-3.05 \Delta+\text { const. }
$$

However Miras in galactic globular clusters fit closely the LMC relation, $(\mathrm{J}-\mathrm{K})=0.56 \log \mathrm{P}+\mathrm{const}$, (cf. Feast et al. 1989, Whitelock et al. 1991). This implies that either equation (2b) is inaccurate or that $\Delta$ is significant and a function of $\mathrm{P}$ (or $\mathrm{z}$ ) (the mass term in equation (3) is very small). In the latter case Miras would be already evolving off the AGB.

I am grateful to Dr P A Whitelock for helpful comments.

\section{REFERENCES}

Feast, M.W. 1981 in Physical Processes in Red Giants ed. I. Iben and A. Renzini (Reidel: Dordrecht) p193.

Feast, M.W. 1989 in The use of Pulsating Stars in Fundamental Problems of Astronomy ed. E.G. Schmidt (Cambridge Univ. Press) p205.

Feast, M.W., Glass, I.S., Whitelock, P.A. and Catchpole, R.M. 1989. Mon. Not. Roy. Astr. Soc. 241, 375.

Feast, M.W. and Whitelock, P.A. 1987 in Late Stages of Stellar Evolution ed. S. Kwok and S.R. Pottasch (Reidel: Dordrecht) p33.

Hartmann, L. and MacGregor, K.B. $1980 . \quad$ Astrophys. J. 242, 260.

Perl, M. and Tuchman, Y. 1990. Astrophys. J. 360, 554.

Pijpers, F.P. $1991 . \quad$ Thesis Leiden.

Pijpers, F.P. and Habing, H.J. 1989. Ast. Astrophys. 215, 334.

Pijpers, F.P. and Hearn, A.G. $1989 . \quad$ Ast. Astrophys. 209, 198.

Renzini, A. and Greggio, L. 1990 in Bulges of Galaxies ESO-CTIO Workshop ed. B.J. Jarvis and D.M. Terndrup 447.

VandenBerg, D.A.and Laskarides, P.G. 1987. Astrophys. J. Sup. 64, 103.

Whitelock, P.A. 1986. Mon. Not. Roy. Ast. Soc. 219, 525.

Whitelock, P.A. 1990 in Confrontation between Stellar Pulsation and Evolution ed. C. Cacciari and G. Clementini (Ast. Soc. Pacif. Conf. Series II).

Whitelock, P.A., Feast, M.W. and Catchpole, R.M. 1991. Mon. Not. Roy. Ast. Soc. 248, 276.

Whitelock, P.A., Pottasch, S.R. and Feast, M.W. 1987 in Late Stages of Stellar Evolution ed. S. Kwok and S.R. Pottasch (Reidel: Dordrecht) p269.

Wood, P.R. 1979. Astrophys. J. 227, 220.

Wood, P.R. 1990 in From Miras to Planetary Nebulae ed. M.O. Mennessier and A Omont (Editions Frontieres) p67.

Wood, P.R., Moore, G.K.G. and Hughes, S.M.G. 1991 in The Magellanic Clouds ed. R. Haynes and D Milne (Kluwer: Dordrecht) p259. 